Monatsschr Kinderheilkd 2009 • 157:14-14

DOI 10.1007/s00112-008-1924-3

Online publiziert: 12. Januar 2009

๑) Springer Medizin Verlag 2009

\author{
F. Zepp ${ }^{1,2}$ \\ ${ }^{1}$ Geschäftsstelle der DGKJ, Berlin \\ 2 Zentrum für Kinder- und Jugendmedizin, \\ Johannes-Gutenberg-Universität Mainz, Mainz
}

\title{
Kompetente Gesundheitsversorgung von Kindern und Jugendlichen
}

\section{Neue DGKJ-Präsidentschaft}

Herr Prof. Dr. Fred Zepp hat zum 01.01.2009 das Amt des Präsidenten der Deutschen Gesellschaft für Kinder- und Jugendmedizin von Herrn Prof. Dr. Hansjosef Böhles übernommen.

Nachdem ich in den vergangenen beiden Jahren Gelegenheit hatte, im Vorstand Eindrücke über die Aufgaben und Verpflichtungen des Präsidenten der Deutschen Gesellschaft für Kinder- und Jugendmedizin (DGKJ) zu sammeln, habe ich am 01.01.2009 das Amt von Herrn Prof. Böhles übernommen. Für die lehrreiche Zeit, den Zuspruch und die Unterstützung auf dem Weg zur Amtsübernahme möchte ich Herrn Prof. Böhles und den Vorstandskollegen/ -innen meinen Dank aussprechen.

Die Ziele unserer Fachgesellschaft sind in der Satzung niedergelegt: Wir wollen die wissenschaftlichen und fachlichen Belange der Kinder- und Jugendmedizin sowie die Belange der Kinder- und Jugendärzte fördern, den Gemeinsinn unter den Mitgliedern pflegen, die wissenschaftlichen Grundlagen für die bestmögliche kinderärztliche Versorgung der Bevölkerung erarbeiten, die internationale Zusammenarbeit auf dem Gebiet der Kinder- und Jugendmedizin pflegen und - „last but not least“ - die Integration pädiatrischer Subspezialitäten fördern. Präziser können die Aufgaben für den Präsidenten und den Vorstand der DGKJ nicht umschrieben werden.

In Zeiten der zunehmenden Ökonomisierung unseres Gesundheitswesens gilt es mehr denn je, Möglichkeiten und Chancen einer kompetenten Gesundheitsversorgung von Kindern und Jugendlichen nachhaltig ins Bewusstsein der Öffentlichkeit, besonders aber in die Wahrnehmung der politischen Entscheidungsträger zu bringen. Kinder- und Jugendmedizin ist in ihren Grundfesten präven- tive Medizin. Aktuelle wissenschaftliche Erkenntnisse unterstreichen die Bedeutung unseres Faches gerade auf dem Gebiet der Prävention. Viele chronische Leiden des Erwachsenen können durch konsequente Nutzung unseres Wissens über Wachstum und Entwicklung zukünftig gelindert oder gar verhindert werden. In den Händen der Pädiatrie liegen die Konzepte für gesünder aufwachsende Generationen durch aktive Prävention und Gesundheitsvorsorge.

\section{( Kinder- und Jugendärzte sind Promotoren für die Gesundheit und Lebensqualität künftiger Generationen}

Der Vorstand und der Präsident werden intensiver als bisher in den Dialog mit Politik und Medien treten, um genau diese Erkenntnis zu vermitteln. Als Dachverband kann die DGKJ dazu beitragen, die Möglichkeiten moderner Kinderund Jugendmedizin verständlich darzustellen, das Bewusstsein der Öffentlichkeit für unsere Konzepte zu schärfen und dadurch letztendlich auch politisch Kraft zu entfalten.

Ohne Frage sind die großen Erfolge der Kinder- und Jugendmedizin auch durch die zunehmende Spezialisierung unserer Subdisziplinen möglich geworden. Eine Entwicklung auf die wir stolz sein dürfen. Wenn wir jedoch die Qualität der Versorgung von Kindern und Jugendlichen auch zukünftig gestalten und verbessern wollen, müssen wir uns heute der Diskussion über die notwendigen Strukturen ggf. auch über die notwendigen Strukturveränderungen stellen. Kinder- und Jugendärzte/-innen sind primär keine Organspezialisten, sondern Spezialisten für das Kind, für den sich kontinuierlich differen- zierenden und verändernden Organismus auf dem Weg in das Erwachsenenalter. Nur mit dem wachen Blick auf dieses verbindende, übergeordnete Konzept werden wir unserer Verantwortung gerecht werden und die Schlagkraft unseres Fachs erhalten und weiter stärken können. Ich sehe es als eine zentrale Aufgabe der Vorstandsarbeit an, den Austausch, besonders aber auch die Zusammenarbeit und den Zusammenhalt zwischen den pädiatrischen Subdisziplinen sowie den Berufsgruppen in Praxis und Klinik zu fördern, $\mathrm{zu}$ gestalten und mit Leben zu füllen.

Kinder- und Jugendärzte/-innen waren und sind Promotoren für die Gesundheit und Lebensqualität künftiger Generationen. Gemeinsam können wir den hohen Versorgungsstandard der Kinder- und Jugendmedizin in Deutschland erhalten und weiterentwickeln. Zusammen mit Ihnen möchte ich alles in meiner Kraft stehende tun, weiterhin die Zielsetzungen unserer Gesellschaft zu verfolgen. Heute danke ich Ihnen für das in mich gesetzte Vertrauen und hoffe auf Ihre aktive Unterstützung in dem Bemühen für die Gesundheit zukünftiger Generationen.

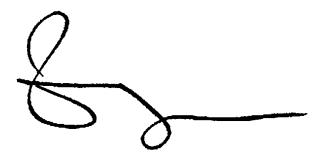

Prof. Dr. F. Zepp

Präsident der Deutschen Gesellschaft für Kinder- und Jugendmedizin

\section{Korrespondenzadresse \\ Prof. Dr. F. Zepp}

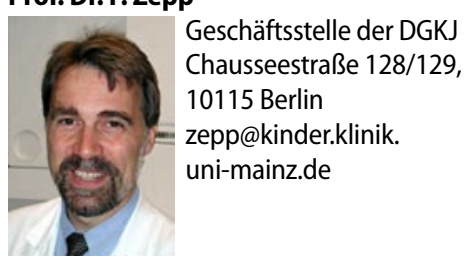

